\title{
REGENERAÇÃO NATURAL EM UMA FLORESTA COM ARAUCÁRIA: INFERÊNCIAS SOBRE O PROCESSO DE CONSTRUÇÃO DA COMUNIDADE DE ESPÉCIES ARBÓREAS
}

\author{
NATURAL REGENERATION IN AN AURACARIA FOREST: INFERENCES ABOUT THE PROCESS \\ OF TREE SPECIES COMMUNITY ASSEMBLAGE
}

\begin{abstract}
Guilherme Neto dos Santos ${ }^{1}$ Pedro Higuchi ${ }^{2}$ Ana Carolina da Silva ${ }^{2}$ Klerysson Julio Farias ${ }^{1}$ Felipe Domingos Machado $^{1}$ Edilaine Duarte ${ }^{3}$ Caroline Fernandes ${ }^{1}$ Francielle Vieira ${ }^{1}$ Rafaela Schröeder Amaral $^{1}$ Vanessa de Aguiar ${ }^{1}$ Felipe Fornara Walter ${ }^{1}$ Bruna Mores ${ }^{1}$ Miguel Angelo Reis ${ }^{1}$
\end{abstract}

\section{RESUMO}

O presente estudo teve como objetivo caracterizar a composição florística do componente regenerante e relacioná-la ao componente arbóreo adulto de um fragmento de Floresta Ombrófila Mista no Sul do Brasil, com o propósito de inferir sobre os processos de construção da comunidade de espécies arbóreas na área. Para isto, foram utilizadas cinco transecções de $100 \times 20 \mathrm{~m}$, alocadas em um fragmento florestal localizado no município de Lages-SC. Cada transecção foi formada por cinco parcelas, distantes $20 \mathrm{~m}$ entre si, subdividas em tamanhos distintos de acordo com a classe de tamanho da planta avaliada. Os componentes regenerante e adulto foram comparados quanto à organização florístico-estrutural (Análise de Variância Multivariada Permutacional e Análise de Espécies Indicadoras), riqueza (rarefação), distribuição espacial dos indivíduos das populações (Índice de Morisita Padronizado) e padrões de coexistência e segregação das espécies nas parcelas (Índice de $C$-score). Os resultados demonstraram diferenças significativas entre adultos e regenerantes em relação à similaridade florístico-estrutural e riqueza. $\mathrm{O}$ componente adulto apresentou maior proporção de populações com distribuição espacial aleatória e menor coexistência de espécies nas parcelas, em relação ao componente regenerante. Infere-se que a construção da comunidade está sujeita a processos ecológicos distintos, cujas intensidades de atuação dependem do estágio ontogenético considerado.

Palavras-chave: Floresta Ombrófila Mista; organização florístico-estrutural; padrões de distribuição espacial; coexistência de espécies.

\section{ABSTRACT}

This study aimed to describe the floristic composition of the regenerative component and to relate it with the adult component of an Araucaria Forest fragment, in South Brazil, in order to infer about the process of tree species community assemblage. To do so, five $10 \times 20 \mathrm{~m}$ transection were allocated, in a forest fragment located at municipality of Lages, SC state. Each transect was made up by five plots, $20 \mathrm{~m}$ from each other, subdivided in different sizes, according to the sampled size class. The adult and regenerative components were compared each other for floristic-structural organization (Permutational Multivariate Analysis of Variance and Indicator Species Analysis), richness (rarefaction), population, individual spatial distribution (Standardized Morisita Index), and pattern of species coexistence or segregation in the studied plots (C-score Index). The results demonstrated significant differences between adults and regenerating

1 Acadêmico de Engenharia Florestal, Departamento de Engenharia Florestal, Universidade do Estado de Santa Catarina, Av. Luiz de Camões, 2090, CEP 88520-000, Lages (SC), Brasil. guineto.florestal@gmail.com / kjfarias. efl@gmail.com / felipe.d.machado@hotmail.com / carolfer9490@gmail.com / franviieira@hotmail.com / rafaelaschroede@gmail.com / vanessa.aguiar89@hotmail.com / felipewalterr@hotmail.com / bruna_mores@ hotmail.com / miguelmoraes.reis@gmail.com

2 Engenheiro Florestal, Dr., Professor do Departamento de Engenharia Florestal, Universidade do Estado de Santa Catarina, Av. Luiz de Camões, 2090, CEP 88520-000, Lages (SC), Brasil. higuchip@gmail.com / carol_sil4@ yahoo.com.br

3 Engenheira Florestal, MSc., Departamento de Engenharia Florestal, Universidade do Estado de Santa Catarina, Av. Luiz de Camões, 2090, CEP 88520-000, Lages (SC), Brasil. ediduarte10@gmail.com

Recebido para publicação em 13/11/2015 e aceito em 11/05/2017

Ci. Fl., v. 28, n. 2, abr. - jun., 2018 
components in relation to floristic-structural similarity and to richness. The adult component presented a greater proportion of populations with a random spatial distribution; and a smaller coexistence of species in plots than the regenerative component. We infer that the tree community assemblage is under influence of different ecological process, which intensity is ontogenetic dependent.

Keywords: Mixed Ombrophyllous Forest; floristic-structural organization; spatial distribution patterns; species coexistence.

\section{INTRODUÇÃO}

A maior parte das florestas de domínio atlântico, incluindo a Floresta Ombrófila Mista (FOM), foi intensamente perturbada e fragmentada para a instalação de práticas agrícolas e pecuárias, reduzindo sua área original. Essas alterações, provavelmente, resultaram em mudanças na sua estrutura e na composição florística (HIGUCHI et al., 2012a). Mesmo com as perturbações que ocorreram, os fragmentos que restaram podem ser mantidos por meio dos processos ecológicos naturais, garantidos pela regeneração natural de espécies arbóreas. Dessa forma, um conhecimento mais profundo sobre o processo de regeneração natural em ambientes florestais naturais é de elevada importância para se determinar a resiliência dos fragmentos e constitui um elemento básico para o planejamento e utilização dos recursos florestais. Segundo Gama, Botelho e Bentesgama (2002), conhecer o "estoque florestal" fornece dados que permitem previsões sobre o comportamento e o desenvolvimento da floresta no futuro.

A regeneração natural caracteriza-se por ser um processo dinâmico, que é essencial para a construção de comunidades de espécies arbóreas (METZ; SOUSA; VALENCIA, 2010) e para o avanço sucessional em um ecossistema florestal (AIDE et al., 2000). Este processo ocorre a partir dos diferentes eventos demográficos, que promovem a entrada (recrutamento) e a saída (mortalidade) de indivíduos de diferentes espécies arbóreas no sistema (WRIGHT et al., 2003). Dentre os fatores ecológicos mais determinantes neste processo, destacam-se os filtros ambientais associados à qualidade do ambiente lumínico e aos solos (WHITMORE, 1989; VIEIRA; SCARIOT, 2006; HIGUCHI et al., 2015), a dispersão de propágulos (NATHAN; MUELLER-LANDAU, 2000) e as várias interações inter e intraespecíficas (competição, predação, herbivoria) (JANZEN, 1970; WHITMORE, 1989; WEBB; PEART, 1999; METZ; SOUSA; VALENCIA, 2010). Neste sentido, dada a natureza complexa das relações existentes entre os componentes bióticos e abióticos em um ecossistema florestal, espera-se elevada heterogeneidade florísticoestrutural ao longo do espaço e do tempo.

Estudos sobre a composição florística e estrutura da regeneração natural, assim como a comparação destes aspectos com o componente arbóreo já estabelecido, são importantes indicadores do potencial de recuperação de ambientes que foram alterados ao longo do tempo por causas naturais ou antrópicas (MARTINS; RODRIGUES, 2002). Para a FOM, vários estudos foram realizados com o propósito de se caracterizar a organização florístico-estrutural do componente regenerativo em condição de sub-bosque, como aqueles realizados nos estados do Rio Grande do Sul (e.g. NARVAES; BRENA; LONGHI 2005; CALLEGARO et al., 2015), Santa Catarina (e.g. CALDATO et al., 1996; MEYER et al., 2013), Paraná (e.g. LIEBSCH; ACRA, 2004) e São Paulo (e.g. POLISEL et al., 2014). De forma geral, os trabalhos demonstraram elevada substituição florística em escala regional e a baixa representatividade de Araucaria angustifolia (Bertol.) Kuntze na regeneração, sugerindo que a mesma apresente limitações de estabelecimento e desenvolvimento em condição de sub-bosque. Porém, poucos trabalhos (e.g. SOUZA et al., 2012; RIBEIRO et al., 2013) contemplaram uma comparação entre os componentes adulto e regenerante, a qual é importante para se entender o processo de desenvolvimento da floresta. Desta forma, o presente estudo teve como objetivo caracterizar a composição florística do componente regenerante e relacionála ao componente arbóreo adulto de um fragmento de Floresta Ombrófila Mista no sul do Brasil, com o propósito de inferir sobre os processos de construção da comunidade de espécies arbóreas na área. Testouse a hipótese de que os componentes apresentam diferenças na composição florístico-estrutural, na riqueza, nos padrões de distribuição espacial das espécies e nos padrões de coexistência das espécies. 


\section{MATERIAL E MÉTODOS}

O presente estudo foi realizado em um fragmento florestal localizado no município de Lages-SC ( $\left.27^{\circ} 51^{\prime} \mathrm{S}, 50^{\circ} 09^{\prime} \mathrm{O}, 1.080 \mathrm{~m}\right)$. O clima da região é temperado úmido, sem estação seca, classificado como $\mathrm{Cfb}$, de acordo com Köppen, com precipitação anual de $1.479,48 \mathrm{~mm}$ e temperatura média anual de $16^{\circ} \mathrm{C}$ (PSCHEIDT et al., 2015). O relevo do local pode ser classificado como predominantemente suave-ondulado e a vegetação original é formada por Floresta Ombrófila Mista e Estepe (IBGE, 2012). A vegetação do fragmento está em estágio tardio de sucessão florestal, embora exista entrada de gado, especialmente mais próximo à borda da floresta, resultando em impacto visível de pisoteio de plântulas, fezes de animais e aberturas de trilhas.

A análise da regeneração natural foi realizada nas cinco transeções de 20x100 m, alocadas por Pscheidt et al. (2015), para avaliação do componente adulto. Essas transecções foram distribuídas no fragmento de forma a contemplar o gradiente de borda existente, estando ao menos $100 \mathrm{~m}$ de distância entre si (Figura 1), e eram subdivididas em 10 parcelas de 10x20 m. Assim, as transecções foram alocadas desde a borda até o interior do fragmento, com a extensão de seu maior comprimento $(100 \mathrm{~m})$ contemplando esse gradiente.

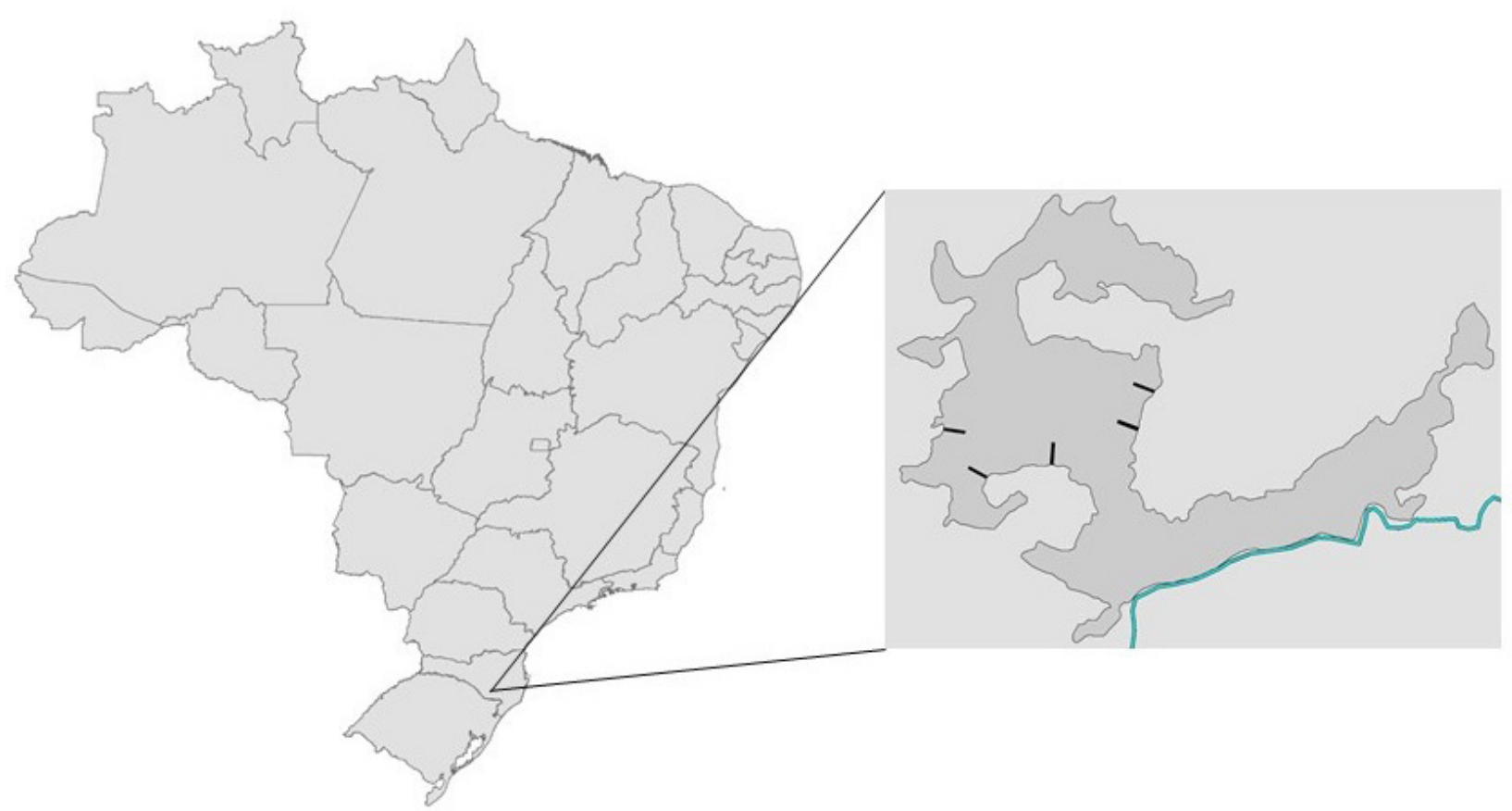

FIGURA 1: Localização das unidades amostrais em um fragmento de Floresta Ombrófila Mista em Lages-SC.

FIGURA 1: Sampling unit locations in an Araucaria Forest fragment in the municipality of Lages, SC state.

Para amostragem da regeneração natural, foram instaladas subparcelas no interior das parcelas situadas nas distâncias de 0, 20, 40,60 e $80 \mathrm{~m}$ da borda, totalizando, assim, cinco subparcelas de regeneração natural em cada transeção e 25 subparcelas no total. As subparcelas foram divididas em função do tamanho das plantas, baseando-se na metodologia proposta por Volpato (1994): classe 1, plantas com altura entre 0,20 e $1 \mathrm{~m}$, amostradas em subparcelas de $5 \mathrm{~m}^{2}(1 \times 5 \mathrm{~m})$; classe 2, plantas com altura maior que $1 \mathrm{~m}$ e até $3 \mathrm{~m}$, amostradas em $10 \mathrm{~m}^{2}(1 \times 10 \mathrm{~m})$; classe 3, plantas com altura maior que $3 \mathrm{~m}$ e DAP (diâmetro a altura do peito, medido a 1,30 do solo) menor que $5 \mathrm{~cm}$, inventariadas em $20 \mathrm{~m}^{2}(1 \times 20 \mathrm{~m})$. No interior de cada unidade amostral, foram identificados e classificados em sua respectiva classe de altura todos os indivíduos regenerantes pertencentes a espécies arbóreas. A identificação foi realizada por especialistas e consulta em literatura e as espécies foram classificadas em famílias organizadas de acordo com o sistema APG IV (ANGIOSPERM PHYLOGENY GROUP, 2016). A determinação da suficiência amostral dos regenerantes foi verificada por meio da construção da curva de acumulação de espécie, por meio do método 
de permutação. O padrão de diversidade desse componente foi determinado por meio do índice de Shannon (H') e da Equabilidade de Pielou (J).

Foi verificada a existência de diferenças na organização florístico-estrutural do componente regenerante em função da setorização borda $(<50 \mathrm{~m})$ e interior $(>50 \mathrm{~m})$, por meio da Análise de Variância Multivariada Permutacional (PERMANOVA). A regeneração natural foi comparada com o componente arbóreo adulto amostrado por Pscheidt et al. (2015), utilizando-se, no entanto, somente as parcelas dos adultos nas quais existiam as subparcelas de regeneração (metade das parcelas utilizadas por Pscheidt et al., 2015), a fim de manter a correspondência para as análises realizadas. Assim, foram utilizadas 25 parcelas de $10 \times 20 \mathrm{~m}$.

A comparação dos valores de riqueza entre os componentes adulto e regenerante foi realizada por meio da técnica de rarefação, considerando, para ambos componentes, a mesma quantidade de indivíduos. A existência de heterogeneidade florístico-estrutural entre adultos e regenerantes foi testada por meio de Análise de Variância Multivariada Permutacional (PERMANOVA). Também foi verificada a associação de espécies com cada um dos componentes por meio da análise de espécies indicadoras (AEI, considerando $p \leq 0,05)$. Para cada componente, foram determinados o número de indivíduos para cada população e o Índice de Morisita para as espécies com mais de cinco indivíduos. Para isso, foi utilizado o Índice de Morisita Padronizado, modificado por Smith-Gill (1975) do Índice de Morisita (MORISITA, 1962). Nele, valores padronizados maiores que 0,5 indicam distribuição espacial agregada, menores que $-0,5$, distribuição uniforme, e valores entre $-0,5$ e 0,5 , distribuição aleatória. Com o propósito de investigar os padrões de coexistência e segregação das espécies nas parcelas, o Índice $C$-score, proposto por Stone e Roberts (1990), foi aplicado para o componente adulto e regenerante. Os valores observados do Índice de $C$-score para ambos os componentes foram comparados com os valores de uma comunidade completamente aleatória, simulados 999 vezes, a partir de um modelo nulo, utilizando-se um algoritmo do tipo swap (GOTELLI; ENTSMINGER, 2003). Valores observados do Índice de $C$-score significativamente maiores do que valores simulados para uma comunidade aleatória indicam espécies com padrão de segregação nas parcelas, ou seja, que tendem a não ocorrerem juntas. Valores observados de $C$-score significativamente menores do que os valores simulados para uma comunidade aleatória indicam que as espécies apresentam um padrão de coexistência nas parcelas. Destaca-se que os adultos e regenerantes foram comparados nas mesmas parcelas, com as mesmas condições ambientais, permitindo, assim, o teste da hipótese de que ambos os componentes apresentam diferenças na composição florístico-estrutural, na riqueza, nos padrões de distribuição espacial das espécies e nos padrões de coexistência das espécies. Os resultados desta análise permitem inferências sobre os processos ecológicos de construção da comunidade, uma vez que os diferentes padrões de coexistência surgem a partir da influência de fatores abióticos (filtros ambientais) e bióticos (interações interespecíficas). Todas as análises foram realizadas por meio da linguagem de programação estatística $\mathrm{R}$ (R DEVELOPMENT CORE TEAM, 2016), com auxílio dos pacotes Vegan (OKSANEN et al., 2016) e LABDSV (ROBERTS, 2016).

\section{RESULTADOS E DISCUSSÃO}

Foram amostrados 698 indivíduos no componente regenerante, pertencentes a 64 espécies, 47 gêneros e 29 famílias (Tabela 1). Apenas quatro indivíduos não foram identificados devido à ausência de folhas no momento da coleta de dados, 10 indivíduos foram identificados somente em nível de família (nove pertencentes a uma espécie de Lauraceae e um a de Myrtaceae) e um em nível de gênero (um indivíduo de Baccharis sp.). A diversidade da comunidade regenerante, caracterizada pelo índice de Shannon, e a dominância ecológica, representada pelo índice de Pielou, apresentaram valores de 3,42 e 0,83, respectivamente, demonstrando elevada diversidade e baixa dominância, o que indica distribuição mais uniforme das espécies em relação ao número de indivíduos amostrados. A curva de acumulação de espécies (Figura 2) demonstrou que a amostragem foi suficiente para a caracterização florística da comunidade, uma vez que com o acréscimo da última parcela ( $4 \%$ do total da área amostrada), ocorreu um incremento de apenas $1,02 \%$ no número de espécies. De acordo com Kersten e Galvão (2011), atinge-se suficiência amostral quando o acréscimo de $10 \%$ em área permite um acréscimo máximo de $5 \%$ de novas espécies amostradas.

Ci. Fl., v. 28, n. 2, abr. - jun., 2018 
Não existiu diferença significativa da organização florístico-estrutural do componente regenerante entre a borda e interior $(p=0,188)$, demonstrando que, para este componente, o efeito de borda não foi evidente. Dentre as possibilidades que podem explicar este resultado, destaca-se o fato da seletividade causada pelo filtro ambiental ser mais evidente em plantas de maior tamanho (HIGUCHI et al., 2015) e/ou a existência de um processo de homogeneização biológica (LÔBO et al., 2011). A riqueza padronizada do componente adulto $(58,95 \pm 0,227)$ foi inferior à observada na comunidade regenerante $(65)$, o que sugere a existência de fatores limitantes ao estabelecimento dos adultos, funcionando como filtro ecológico (GANDOLFI; JOLY; RODRIGUES, 2007; HIGUCHI et al., 2015).

TABELA 1: Composição florística, número de indivíduos (N) por componente (regenerante e adulto), Índice de Morisita Padronizado (IMP, sendo G = agregada e L = aleatória) para cada espécie, Índice de $C$-score, riqueza determinada por rarefação e espécies indicadoras $\left({ }^{1}\right)$ para os estratos regenerantes e adultos, em um fragmento de Floresta Ombrófila Mista em Lages-SC.

TABLE 1: Floristic composition, number of individuals (N) by component (regenerative and adult), Standardized Morisita Index (IMP, $\mathrm{G}=$ aggregated and $\mathrm{L}=$ random) for each species, C-score Index, richness determined by rarefaction and indicator species $\left({ }^{1}\right)$ for regenerative and adult components, in an Araucaria Forest, in the municipality of Lages, SC state.

\begin{tabular}{|c|c|c|c|c|c|}
\hline \multirow{2}{*}{ Famílias } & \multirow{2}{*}{ Espécies } & \multicolumn{2}{|c|}{ Regenerantes } & \multicolumn{2}{|c|}{ Adultos } \\
\hline & & $\mathrm{N}$ & IMP & $\mathrm{N}$ & IMP \\
\hline \multirow{5}{*}{ Anacardiaceae } & Lithrea brasiliensis Marchand & 3 & - & $62^{1}$ & $0,5023^{\mathrm{G}}$ \\
\hline & Schinus lentiscifolius Marchand & - & - & 1 & - \\
\hline & Schinus polygama (Cav.) & 1 & - & - & - \\
\hline & Cabrera & 1 & - & - & - \\
\hline & Schinus terebinthifolius Raddi & - & - & 5 & - \\
\hline Annonaceae & $\begin{array}{l}\text { Annona rugulosa (Schltdl.) H. } \\
\text { Rainer }\end{array}$ & 6 & $0,1085^{\mathrm{L}}$ & 4 & - \\
\hline \multirow{3}{*}{ Aquifoliaceae } & Ilex dumosa Reissek & 2 & - & 3 & - \\
\hline & Ilex microdonta Reissek & 6 & $0,5222^{\mathrm{G}}$ & 8 & $0,5291^{\mathrm{G}}$ \\
\hline & Ilex theezans Mart. ex Reissek & 3 & - & 12 & $0,4556^{\mathrm{L}}$ \\
\hline Araliaceae & Oreopanax fulvus Marchal & 1 & - & - & - \\
\hline Araucariaceae & $\begin{array}{l}\text { Araucaria angustifolia (Bertol.) } \\
\text { Kuntze }\end{array}$ & 19 & $0,5010^{\mathrm{G}}$ & $77^{1}$ & $0,2950^{\mathrm{L}}$ \\
\hline \multirow{4}{*}{ Asteraceae } & Baccharis sp. & 1 & - & - & - \\
\hline & $\begin{array}{l}\text { Dasyphyllum tomentosum } \\
\text { (Spreng.) Cabrera }\end{array}$ & $17^{1}$ & $0,5173^{\mathrm{G}}$ & - & - \\
\hline & $\begin{array}{l}\text { Gochnatia polymorpha (Less.) } \\
\text { Cabrera }\end{array}$ & - & - & 3 & - \\
\hline & $\begin{array}{l}\text { Vernonanthura discolor } \\
\text { (Spreng.) H. Rob. }\end{array}$ & 1 & - & $16^{1}$ & $0,4271^{\mathrm{L}}$ \\
\hline Berberidaceae & Berberis laurina Billb. & 1 & - & - & - \\
\hline Bignoniaceae & Jacaranda puberula Cham. & 8 & $0,5086^{\mathrm{G}}$ & 37 & $0,5366^{\mathrm{G}}$ \\
\hline \multirow{2}{*}{ Celastraceae } & Maytenus boaria Molina & 1 & - & 3 & - \\
\hline & Maytenus dasyclados Mart. & 5 & - & 8 & $-0,0323^{\mathrm{L}}$ \\
\hline Clethraceae & Clethra scabra Pers. & 2 & - & $16^{1}$ & $0,5058^{\mathrm{G}}$ \\
\hline Cunoniaceae & Lamanonia ternata Vell. & 11 & $0,5447^{\mathrm{G}}$ & 14 & $0,5004^{\mathrm{G}}$ \\
\hline Dicksoniaceae & Dicksonia sellowiana Hook. & - & - & 25 & $0,6700^{\mathrm{G}}$ \\
\hline Erythroxylaceae & $\begin{array}{l}\text { Erythroxylum deciduum A.St.- } \\
\text { Hil. }\end{array}$ & - & - & 2 & - \\
\hline \multirow{3}{*}{ Euphorbiaceae } & $\begin{array}{l}\text { Sapium glandulosum (L.) } \\
\text { Morong }\end{array}$ & - & - & $9^{1}$ & $0,2820^{\mathrm{L}}$ \\
\hline & Sebastiana brasiliensis Spreng. & 3 & - & 7 & $1,0000^{\mathrm{G}}$ \\
\hline & $\begin{array}{l}\text { Sebastiania commersoniana } \\
\text { (Baill.) L.B. Sm. \& Downs }\end{array}$ & 2 & - & 9 & $0,7169^{\mathrm{G}}$ \\
\hline \multirow{3}{*}{ Fabaceae } & $\begin{array}{l}\text { Dalbergia frutescens (Vell.) } \\
\text { Britton }\end{array}$ & 3 & - & 7 & $0,5280^{\mathrm{G}}$ \\
\hline & $\begin{array}{l}\text { Machaerium paraguariense } \\
\text { Hassl. }\end{array}$ & - & - & 3 & - \\
\hline & Mimosa scabrella Benth. & 2 & - & - & - \\
\hline \multirow{4}{*}{ Lauraceae } & $\begin{array}{l}\text { Cinnamomum amoenum (Nees) } \\
\text { Kosterm. }\end{array}$ & 1 & - & 3 & - \\
\hline & Lauraceae sp. & 9 & $0,5754^{\mathrm{G}}$ & - & - \\
\hline & Ocotea puberula (Rich.) Nees & 11 & $0,5852^{\mathrm{G}}$ & - & - \\
\hline & Ocotea pulchella Mart. & 25 & $0,5309^{\mathrm{G}}$ & 14 & $-0,0985^{\mathrm{L}}$ \\
\hline
\end{tabular}


TABELA 1: Continuação...

TABLE 1: Continued...

\begin{tabular}{|c|c|c|c|c|c|}
\hline \multirow{2}{*}{ Famílias } & \multirow{2}{*}{ Espécies } & \multicolumn{2}{|c|}{ Regenerantes } & \multicolumn{2}{|c|}{ Adultos } \\
\hline & & $\mathrm{N}$ & IMP & $\mathrm{N}$ & IMP \\
\hline Loganiaceae & $\begin{array}{l}\text { Strychnos brasiliensis (Spreng.) } \\
\text { Mart. }\end{array}$ & - & - & 2 & - \\
\hline \multirow{3}{*}{ Melastomataceae } & Leandra regnellii (Triana) Cogn. & $27^{1}$ & $0,5801^{\mathrm{G}}$ & - & - \\
\hline & Miconia cinerascens Miq. & $23^{1}$ & $0,4867^{\mathrm{L}}$ & - & - \\
\hline & $\begin{array}{l}\text { Miconia hyemalis A. St.-Hil. \& } \\
\text { Naudin }\end{array}$ & 2 & - & - & - \\
\hline \multirow{18}{*}{ Myrtaceae } & Acca sellowiana (O.Berg) Burret & - & - & 6 & $0,3797^{\mathrm{L}}$ \\
\hline & $\begin{array}{l}\text { Blepharocalyx salicifolius } \\
\text { (Kunth) O. Berg }\end{array}$ & 64 & $0,5370^{\mathrm{G}}$ & 23 & $0,5187^{\mathrm{G}}$ \\
\hline & Calyptranthes concinna DC. & 33 & $0,4379^{\mathrm{L}}$ & 49 & $0,5004^{\mathrm{G}}$ \\
\hline & $\begin{array}{l}\text { Campomanesia xanthocarpa } \\
\text { Mart. ex O. Berg }\end{array}$ & 4 & - & - & - \\
\hline & Eugenia pluriflora DC. & 7 & $-0,2587^{\mathrm{L}}$ & 11 & $-0,0392^{\mathrm{L}}$ \\
\hline & Eugenia uniflora $\mathrm{L}$. & 1 & - & - & - \\
\hline & Eugenia uruguayensis Cambess. & 2 & - & - & - \\
\hline & $\begin{array}{l}\text { Myrceugenia euosma }(\mathrm{O} . \mathrm{Berg}) \\
\text { D. Legrand }\end{array}$ & 1 & - & 1 & - \\
\hline & $\begin{array}{l}\text { Myrceugenia glaucescens } \\
\text { (Cambess.) D. Legrand \& } \\
\text { Kausel }\end{array}$ & $74^{1}$ & $0,5197^{\mathrm{G}}$ & 1 & - \\
\hline & $\begin{array}{l}\text { Myrceugenia myrcioides } \\
\text { (Cambess.) O. Berg }\end{array}$ & 1 & - & - & - \\
\hline & $\begin{array}{l}\text { Myrceugenia oxysepala (Burret) } \\
\text { D. Legrand \& Kausel }\end{array}$ & 3 & - & 3 & - \\
\hline & Myrcia guianensis (Aubl.) DC. & 5 & - & $21^{1}$ & $0,2789^{\mathrm{L}}$ \\
\hline & Myrcia hartwegiana D. Legrand & $19^{1}$ & $0,5358^{\mathrm{G}}$ & 1 & - \\
\hline & Myrcia hatschbachii D. Legrand & 2 & - & - & - \\
\hline & $\begin{array}{l}\text { Myrcia selloi (Spreng.) N. } \\
\text { Silveira }\end{array}$ & 13 & $0,5627^{\mathrm{G}}$ & 6 & $0,3797^{\mathrm{L}}$ \\
\hline & Myrcia palustris DC. & 30 & $0,5409^{\mathrm{G}}$ & 42 & $0,5133^{\mathrm{G}}$ \\
\hline & $\begin{array}{l}\text { Myrrhinium atropurpureum } \\
\text { Schott }\end{array}$ & $21^{1}$ & $0,5183^{\mathrm{G}}$ & 1 & - \\
\hline & Myrtaceae sp.1 & 1 & - & - & - \\
\hline Podocarpaceae & $\begin{array}{l}\text { Podocarpus lambertii Klotzsch } \\
\text { ex Endl. }\end{array}$ & 14 & $0,5124^{\mathrm{G}}$ & $44^{1}$ & $0,5127^{\mathrm{G}}$ \\
\hline \multirow{3}{*}{ Primulaceae } & $\begin{array}{l}\text { Myrsine coriacea (Sw.) R. Br. } \\
\text { ex Roem. \& Schult. }\end{array}$ & 10 & $0,3580^{\mathrm{L}}$ & 3 & - \\
\hline & $\begin{array}{l}\text { Myrsine lorentziana }(\mathrm{Mez}) \\
\text { Arechav. }\end{array}$ & 74 & $0,5084^{\mathrm{G}}$ & 47 & $0,5241^{\mathrm{G}}$ \\
\hline & Myrsine umbellata Mart. & - & - & 6 & $0,1085^{\mathrm{L}}$ \\
\hline Proteaceae & Roupala montana Aubl. & 2 & - & 1 & - \\
\hline Quillajaceae & $\begin{array}{l}\text { Quillaja brasiliensis (A.St.-Hil. } \\
\text { \& Tul.) Mart. }\end{array}$ & - & - & 1 & - \\
\hline \multirow{2}{*}{ Rhamnaceae } & Rhamnus sphaerosperma $\mathrm{Sw}$. & 2 & - & - & - \\
\hline & Scutia buxifolia Reissek & 2 & - & 9 & $0,2820^{\mathrm{L}}$ \\
\hline Rosaceae & Prunus myrtifolia (L.) Urb. & 18 & $0,5189^{\mathrm{G}}$ & 8 & $-0,0323^{\mathrm{L}}$ \\
\hline \multirow[t]{2}{*}{ Rutaceae } & $\begin{array}{l}\text { Zanthoxylum kleinii } \\
\text { (R.S.Cowan) P.G.Waterman }\end{array}$ & - & 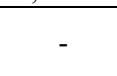 & $15^{1}$ & $-0,3161^{\mathrm{L}}$ \\
\hline & Zanthoxylum rhoifolium Lam. & 5 & - & 6 & $-0,2155^{\mathrm{L}}$ \\
\hline \multirow{3}{*}{ Salicaceae } & Banara tomentosa Clos & 3 & - & 1 & - \\
\hline & Casearia decandra Jacq. & 26 & $0,3755^{\mathrm{L}}$ & 38 & $0,2090^{\mathrm{L}}$ \\
\hline & $\begin{array}{l}\text { Xylosma ciliatifolia (Clos) } \\
\text { Eichler }\end{array}$ & 2 & - & 2 & - \\
\hline
\end{tabular}

Continuação... 
TABELA 1: Continuação...

TABLE 1: Continued...

\begin{tabular}{|c|c|c|c|c|c|}
\hline \multirow{2}{*}{ Famílias } & \multirow{2}{*}{ Espécies } & \multicolumn{2}{|c|}{ Regenerantes } & \multicolumn{2}{|c|}{ Adultos } \\
\hline & & $\mathrm{N}$ & IMP & $\mathrm{N}$ & IMP \\
\hline \multirow[t]{3}{*}{ Sapindaceae } & $\begin{array}{l}\text { Allophylus edulis (A. St.-Hil., A. } \\
\text { Juss. \& Cambess.) Hieron. ex } \\
\text { Niederl. }\end{array}$ & 10 & $0,5764^{\mathrm{G}}$ & 10 & $0,5016^{\mathrm{G}}$ \\
\hline & Cupania vernalis Cambess. & 2 & - & - & - \\
\hline & Matayba elaeagnoides Radlk. & $23^{1}$ & $0,4867^{\mathrm{L}}$ & 10 & $0,6387^{\mathrm{G}}$ \\
\hline Solanaceae & $\begin{array}{l}\text { Solanum sanctaecatharinae } \\
\text { Dunal }\end{array}$ & - & - & 1 & - \\
\hline Styracaceae & Styrax leprosus Hook. \& Arn. & 1 & - & 2 & - \\
\hline \multirow{2}{*}{ Symplocaceae } & $\begin{array}{l}\text { Symplocos tetrandra (Mart.) } \\
\text { Benth. }\end{array}$ & - & - & 1 & - \\
\hline & $\begin{array}{l}\text { Symplocos uniflora (Pohl) } \\
\text { Benth. }\end{array}$ & 6 & $0,8009^{\mathrm{G}}$ & 5 & - \\
\hline Thymelaeaceae & Daphnopsis racemosa Griseb. & 2 & - & - & - \\
\hline Verbenaceae & Duranta vestita Cham. & 6 & $0,6416^{\mathrm{G}}$ & 17 & $0,5412^{\mathrm{G}}$ \\
\hline Winteraceae & Drimys brasiliensis Miers & 9 & $0,5282^{\mathrm{G}}$ & 22 & $0,3521^{\mathrm{L}}$ \\
\hline Abundância Total & & 698 & - & 768 & - \\
\hline Riqueza padronizada $(\mathrm{n}=764)$ & & 65 & & 58,95 & $(0,227)$ \\
\hline C-score observado & & 20,12 & $p=0,86$ & 21,03 & $p=0,05$ \\
\hline
\end{tabular}

Valores de $p$ indicam a significância das diferenças entre os valores observados de $C$-score e os valores médios simulados $(\mathrm{n}=999)$ para uma comunidade com estruturação completamente aleatória. Valor entre parênteses indicam o erro padrão para o número de espécies determinado por rarefação.

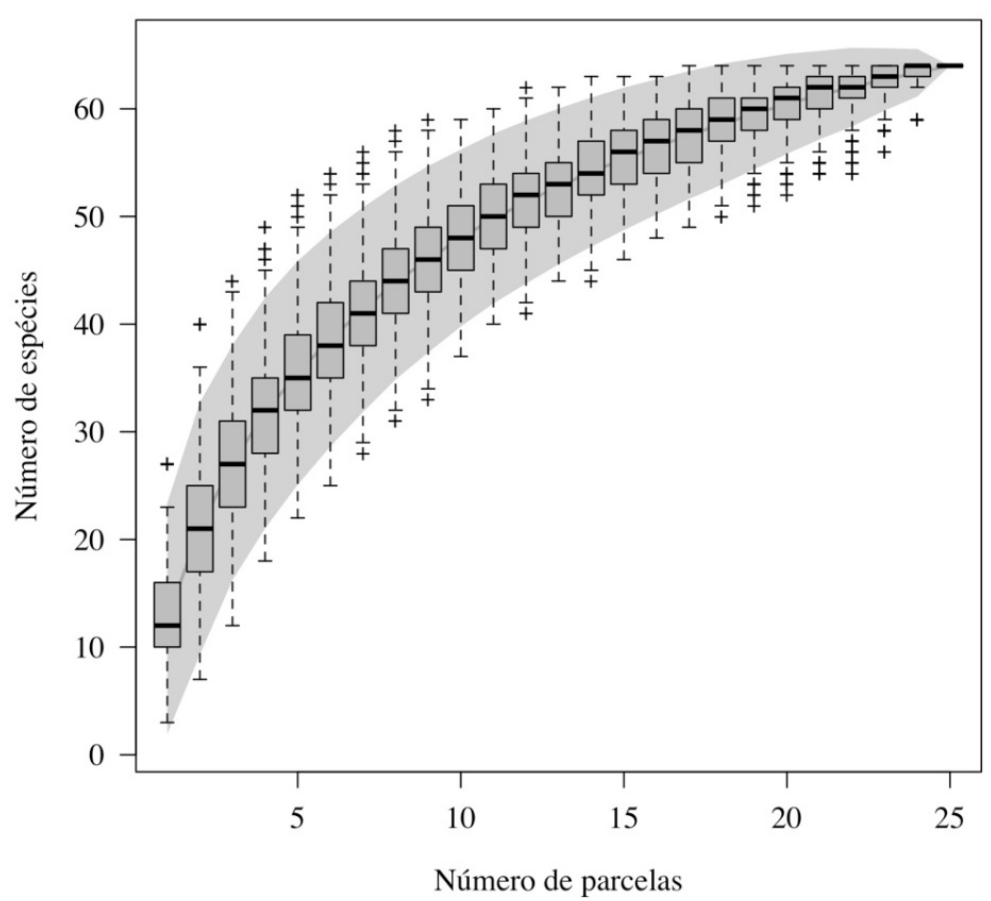

FIGURA 2: Curva de acumulação de espécies do componente regenerativo arbóreo em um fragmento de Floresta Ombrófila Mista em Lages-SC.

FIGURE 2: Species accumulation curve of regenerative tree species component in an Araucaria Forest fragment, in the municipality of Lages, SC state. 
A família de maior riqueza para os regenerantes foi Myrtaceae (17 espécies), seguida por Lauraceae (quatro), Aquifoliaceae, Asteraceae, Melastomataceae, Salicaceae e Sapindaceae, essas últimas com três espécies cada. Os gêneros de maior riqueza foram Myrcia (cinco espécies), Myrceugenia (quatro), Eugenia (três) e Ilex (três). Para o componente adulto, cuja caracterização florístico-estrutural foi apresentada de forma detalhada por Pscheidt et al. (2015), em 25 parcelas foram observados 768 indivíduos pertencentes a 59 espécies, 44 gêneros e 31 famílias, com maior riqueza de famílias (Myrtaceae, Anacardiaceae, Aquifoliaceae, Euphorbiaceae, Primulaceae e Salicaceae) e gêneros (Myrcia, Ilex, Myrceugenia e Myrsine) semelhantes ao do componente regenerante. O padrão de famílias e gêneros se assemelha ao encontrado em outros estudos em FOM da região sul do Brasil (e.g. NASCIMENTO; LONGHI; BRENA, 2001; RONDON NETO; WATZLAWICK; CALDEIRA, 2001; PAISE; VIEIRA, 2005; SILVA et al., 2012; HIGUCHI et al., 2013; MARCON et al., 2014; NEGRINI et al., 2014; SANTOS et al., 2015), tanto para os regenerantes quanto para os adultos. Por outro lado, estudos realizados na região sudeste do Brasil em áreas de FOM (e.g. SOUZA et al., 2012; RIBEIRO et al., 2013; POLISEL et al., 2014) demonstraram diferenças, como, por exemplo, na elevada representatividade de Rubiaceae no sub-bosque. Estas variações regionais sugerem que a composição florística do componente regenerativo em FOM, assim como o componente arbóreo adulto, seja parcialmente determinada pelo conjunto regional de espécies, condicionada por fatores climáticos (e.g. temperatura) e históricos (e.g. rotas de migração) (HIGUCHI et al., 2012b; OLIVEIRA FILHO et al., 2015). Este aspecto deve ser considerado em programas de restauração ecológica, principalmente no que se refere à seleção de espécies.

Apesar da semelhança quanto à representatividade de famílias e gêneros, os componentes adulto e regenerante difeririam em relação à composição florístico-estrutural, como indicado pela PERMANOVA $(p=0,001)$ e AEI. As espécies indicadoras do componente adulto (Tabela 1) foram aquelas abundantes no dossel, porém, com baixa capacidade de se estabelecerem no sub-bosque da floresta, possivelmente por apresentarem limitações ecológicas para a germinação e desenvolvimento e/ou baixa produção de propágulos. Já as indicadoras do componente regenerante foram os táxons que, apesar de estarem bem representados no sub-bosque, estiveram ausentes ou apresentaram poucos indivíduos no dossel da floresta por diferentes motivos, podendo ser: i) apresentam alguma limitação ecológica para o desenvolvimento, devido a fatores ambientais ou bióticos (herbivoria, competição, etc.); ii) estão em fase inicial de colonização na área estudada ou são tolerantes às perturbações existentes na área, como, por exemplo, o pastoreio (SAMPAIO; GUARINO, 2007), sendo que poderão alcançar o dossel com o tempo; ou iii) são espécies de pequeno porte, que raramente alcançam um DAP maior do que $5 \mathrm{~cm}$.

A elevada heterogeneidade florístico-estrutural entre adultos e regenerantes foi o mesmo padrão observado por Leyser et al. (2012) em uma floresta subtropical na região do Alto-Uruguai, sendo que as diferentes exigências das espécies quanto à disponibilidade de luz foram destacadas pelos autores como um importante fator. Souza et al. (2012), também em área de Floresta Ombrófila Mista, observaram uma similaridade entre os componentes de apenas 0,28 pelo índice de Jaccard, o que sugere que o particionamento florístico ao longo do perfil vertical da floresta seja comum.

Destacam-se, também, aquelas espécies com elevada representatividade em ambos os componentes, demonstrando uma boa capacidade de estabelecimento e de manutenção nas condições atuais da floresta, que são candidatas para serem utilizadas em plantios de adensamento e enriquecimento no sub-bosque, como, por exemplo, Blepharocalyx salicifolius (Kunth) O. Berg (64 indivíduos regenerantes e 23 adultos), Calyptranthes concinna DC. (33 regenerantes e 49 adultos), Myrcia palustris DC. (30 regenerantes e 42 adultos), Myrsine lorentziana (Mez) Arechav. (74 regenerantes e 47 adultos) e Casearia decandra Jacq. (26 regenerantes e 38 adultos).

Em relação à distribuição espacial das espécies com mais do que cinco indivíduos (Tabela 1), nota-se que a maioria dos regenerantes $(75,86 \%, 22$ espécies) apresentou distribuição agregada, com o restante $(24,14 \%$, sete espécies) com um padrão de distribuição aleatória. A predominância do padrão de distribuição espacial agregado também foi observada por Kanieski et al. (2012) para o componente regenerativo em uma área de FOM no Rio Grande do Sul. Para o componente adulto, observa-se que os padrões de distribuição agregada e aleatória ocorreram em mesma proporção, com 17 espécies cada. Em um fragmento de Floreta Ombrófila Mista em Lages, Negrini et al. (2012) também observaram uma expressiva participação de espécies com distribuição aleatória no componente adulto. Do componente regenerativo 
para o adulto, apenas sete espécies apresentaram mudanças no padrão de distribuição espacial, sendo que cinco (Araucaria angustifolia, Ocotea pulchella Mart., Myrcia laruotteana Cambess., Prunus myrtifolia (L.) Urb. e Drimys brasiliensis Miers) passaram de agregado na regeneração para aleatório no adulto, e duas (Calyptranthes concinna DC. e Matayba elaeagnoides Radlk.) passaram de aleatório para agregado no adulto.

A forma como as árvores ocupam o espaço horizontal em uma floresta, definindo, assim, o padrão de distribuição espacial das espécies, representa o resultado de vários processos ecológicos que atuam ao longo do tempo. Durante o ciclo de vida de uma planta, a dispersão de propágulos é considerada como o primeiro evento espacialmente estruturado, podendo apresentar uma variação em função das diferentes síndromes existentes (zoocoria, anemocoria e autocoria) (NATHAN; MUELLER-LANDAU, 2000). Após a dispersão, da germinação até o estágio adulto, os indivíduos arbóreos ficam sujeitos a filtros ecológicos de natureza biótica (e.g., competição, herbivoria e predação) e abiótica (condições ambientais e eventos estocásticos) (JANZEN, 1970; WHITMORE, 1989; SCHNITZER; DALLING; CARSON, 2000; GANDOLFI; JOLY; RODRIGUES, 2007; HIGUCHI et al., 2015), que ocorrem com diferentes padrões de estruturação espacial. Neste sentido, o padrão de distribuição agregado pode ser o resultado de uma dispersão espacialmente limitada a partir de árvores-matrizes e/ou das exigências ecológicas por condições ambientais específicas que ocorrem na forma de manchas (e.g. clareiras, umidade do solo, tipo de solo). Por outro lado, uma distribuição aleatória pode ser o resultado de uma dispersão mais irregular e/ou interações ecológicas (e.g. competição inter e intraespecífica, predação e herbivoria). Ressalta-se que, enquanto que a influência de filtros ecológicos é mais evidente em estágios ontogenéticos mais maduros (JABOT; ETIENNE; CHAVE, 2008; HIGUCHI et al., 2015), espera-se que a influência da dispersão de propágulos seja mais perceptível na regeneração natural, que representa as primeiras fases de vida das plantas (HIGUCHI et al., 2015). Desta forma, como a maior proporção das espécies regenerantes apresenta uma distribuição espacial agregada, infere-se que, na área estudada, a maioria das espécies que ocorrem neste componente apresente uma dispersão de propágulos espacialmente limitada. Por outro lado, seguindo o mesmo raciocínio, os padrões espaciais observados para o componente adulto seriam o resultado de exigências ecológicas a condições ambientais específicas, resultando em padrão agregado, e de interações inter e intraespecíficas (e.g., competição, herbivoria), definindo um padrão aleatório. Neste sentido, as cinco espécies que passaram de uma distribuição agregada para aleatória, do componente regenerativo para o adulto, podem ser considerados exemplos da ação de eventos biológicos promotores da mortalidade dependente de densidade (e.g., competição; herbivoria), provocando, assim, a mudança no padrão espacial (JANZEN, 1970; GAVIN; PEART, 1997).

Além de uma distribuição espacial, predominantemente, agregada, as espécies que compõem a comunidade regenerante não apresentaram nenhum padrão de coexistência ou segregação entre si ( $C$-score observado $=20,12 ; p=0,86$ ), ou seja, a organização da comunidade não diferiu de uma comunidade organizada de forma aleatória, gerada por meio de um modelo nulo $(C$-score modelo nulo $=20,16)$. Por outro lado, o componente adulto, além de apresentar um maior percentual de espécies com distribuição espacial aleatória, apresentou um padrão de segregação das espécies $(C$-score $=21,03 ; p=0,05)$, ou seja, de baixa coexistência das espécies nas parcelas, diferindo do observado para uma comunidade gerada por modelo nulo $(C$-score modelo nulo $=20,16)$. Da mesma forma que os padrões espaciais, os padrões de coexistência ou segregação das espécies também resultam de vários fatores ecológicos que atuam durante a construção das comunidades biológicas, como, por exemplo, eventos estocásticos, interações bióticas, dispersão e condições ambientais (CARUSO; POWELL; RILLIG, 2012; GÖTZENBERGER et al., 2012; CAPITÁN; CUENDA; ALONSO, 2015). Desta forma, a partir dos resultados encontrados, é possível inferir que o processo de construção da comunidade regenerante avaliada foi caracterizado por uma dispersão de propágulos espacialmente limitada, gerando uma comunidade com espécies distribuídas, predominantemente, de forma agregada, porém, sem um padrão evidente de organização de coexistência. Com o tempo, esta comunidade regenerante, ao passar pela ação de condicionantes ecológicos (e.g., competição, filtros ambientais), irá resultar em um componente adulto no qual a maior proporção de espécies apresenta uma distribuição aleatória, com tendência de não coexistirem nas mesmas parcelas. 


\section{CONCLUSÕES}

As hipóteses testadas foram confirmadas, demonstrando que os componentes adulto e regenerante em um fragmento de Floresta Ombrófila Mista, em avançado estágio sucessional, apresentaram distinção no que se refere à composição florístico-estrutural (matrizes de abundância de espécies), riqueza, padrões de distribuição espacial e de coexistência das espécies. Destaca-se que ambos os componentes foram avaliados ao longo do perfil vertical das mesmas parcelas, estando, portanto, sujeitas às mesmas condições ambientais. Além disso, não foi observada a influência significativa da setorização de borda e interior sobre a organização do componente regenerativo. Dessa forma, o presente estudo demonstrou a importância de se contemplar classes de tamanhos inferiores em estudos com espécies arbóreas em florestas naturais, pois, desta forma, é possível uma visão mais completa sobre como ocorre a construção de comunidades de árvores.

\section{AGRADECIMENTOS}

Ao $\mathrm{CNPq}$, pela bolsa de Iniciação Científica concedida ao primeiro autor e pelas bolsas de produtividade concedidas ao segundo e terceiro autores.

\section{REFERÊNCIAS}

AIDE, T. M. et al. Forest regeneration in a chronosequence of tropical abandoned pastures: implications for restoration ecology. Restoration ecology, Hoboken, v. 8, n. 4, p. 328-338, 2000.

ANGIOSPERM PHYLOGENY GROUP. An update of the Angiosperm Phylogeny Group classification for the orders and families of flowering plants: APG IV. Botanical Journal of the Linnean Society, Londres, v. 181, n. 1, p. 1-20, 2016.

CALDATO, S. L. et al. Estudo da regeneração natural, banco de sementes e chuva de sementes na Reserva Genética Florestal de Caçador, SC. Ciência Florestal, Santa Maria, v. 6, n.1, p. 27-38, 1996.

CALLEGARO, R. M. et al. Regeneração natural de espécies arbóreas em diferentes comunidades de um remanescente de Floresta Ombrófila Mista, Ciência Rural, Santa Maria, v. 45, n. 10, 1795-1801, 2015.

CAPITÁN, J. A.; CUENDA, S.; ALONSO, D. How similar can co-occurring species be in the presence of competition and ecological drift? Journal of the Royal Society Interface, London, v. 12, n. 110, 2015. CARUSO, T; POWELL, J. R.; RILLIG, M. C. Compositional divergence and convergence in local communities and spatially structured landscapes. PloSone, San Francisco, v. 7, n. 4, p. e35942, 2012.

GAMA, J. R. V.; BOTELHO, S. A.; BENTESGAMA, M. M. Composição florística e Estrutura da regeneração natural de floresta secundária de Várzea Baixa no Estuário Amazônico. Revista Árvore, Viçosa, MG, v. 26, n. 5, p. 559-566, 2002.

GANDOLFI, S.; JOLY, C. A.; RODRIGUES, R. R. Permeability-impermeability: canopy trees as biodiversity lter. Sciencia Agricola, Piracicaba, v. 64, n. 4, p. 433-438, 2007.

GAVIN, D. G.; PEART, D. R. Spatial structure and regeneration of Tretamerista glabra in peat swamp rain forest in Indonesian Borneo. Plant Ecology, New York, v. 131, n. 2, p. 223-231, 1997.

GOTELLI, N. J.; ENTSMINGER, N. J. Swap algorithms in null model analysis. Ecology, Hoboken, v. 84, n. 2, p. 532-535, 2003.

GÖTZENBERGER, L. et al. Ecological assembly rules in plant communities-approaches, patterns and prospects. Biological Reviews, Hoboken, v. 87, n. 1, p. 111-127, 2012.

HIGUCHI, P. et al. Fatores determinantes da regeneração natural em um fragmento de floresta com araucária no planalto catarinense. Scientia Forestalis, Piracicaba, v. 43, n. 106, p. 251-259, 2015.

HIGUCHI, P. et al. Florística e estrutura do componente arbóreo e análise ambiental de um fragmento de Floresta Ombrófila Mista Alto-Montana no município de Painel, SC. Ciência Florestal, Santa Maria, v. 23, n. 1, p. 153-164, 2013.

HIGUCHI, P. et al. Influência de variáveis ambientais sobre o padrão estrutural e florístico do componente arbóreo, em um fragmento de Floresta Ombrófila Mista Montana em Lages, SC. Ciência Florestal, Santa Maria, v. 22, n. 1, p. 79-90, 2012a. 
HIGUCHI, P. et al. Floristic composition and phytogeography of the tree component of Araucaria Forest fragments in southern Brazil. Brazilian Journal of Botany, São Paulo, v. 35, n. 2, p. 145-157, 2012b. IBGE. Manual técnico da vegetação brasileira. Rio de Janeiro: Fundação IBGE, 2012. 271 p.

JABOT, F.; ETIENNE, R. S.; CHAVE, J. Reconciling neutral community models and environmental filtering: theory and empirical test. Oikos, Hoboken, v. 117, n. 9, p. 1308-1320, 2008.

JANZEN, D. H. Herbivores and the number of tree species in tropical forests. The American naturalist, Chicago, v. 104, n. 940, p. 501-528, 1970.

KANIESKI, M. R. et al. Diversidade e padrões de distribuição espacial de espécies no estágio de regeneração natural em São Francisco de Paula, RS, Brasil. Ciência Florestal, Santa Maria, v. 42, n.3, p. 509-518, 2012. KERSTEN, R. A.; GALVÃO, F. Suficiência amostral em inventários florísticos e fitossociológicos. In: FELFILI, J. M. et al. (Org.). Fitossociologia no Brasil: métodos e estudos de casos. Viçosa, MG: Editora UFV, 2011. p. 156-173.

LEYSER, G. et al. Regeneração de espécies arbóreas e relações com componente adulto em uma floresta estacional no vale do rio Uruguai, Brasil. Acta Botanica Brasilica, Belo Horizonte, v. 26, n. 1, p. 74-83, 2012.

LIEBSCH, D.; ACRA, L. A. Riqueza de espécies arbóreas de sub-bosque de um fragmento de Floresta Ombrófila Mista em Tijucas do Sul, PR. Ciência Florestal, Santa Maria, v. 14, n. 1, p. 67-76, 2004. LÔBO, D. et al. Forest fragmentation drives Atlantic forest of northeastern Brazil to biotic homogenization. Diversity and Distributions, Hoboken, v. 17, n. 2, p. 287-296, 2011.

MARCON, A. K. et al. Variação florístico-estrutural em resposta à heterogeneidade ambiental em uma Floresta Nebular em Urubici, Planalto Catarinense. Scientia Forestalis, Piracicaba, v. 42, n. 103, p. 439-450, 2014.

MARTINS, S. V.; RODRIGUES, R. R. Gap-phase regeneration in a semideciduous mesophytic forest, south-eastern Brazil. Plant Ecology, New York, v. 163, n. 1, p. 51-62, 2002.

METZ, M. R.; SOUSA, W. P.; VALENCIA, R. Widespread density-dependent seedling mortality promotes species coexistence in a highly diverse Amazonian rain forest. Ecology, Hoboken, v. 91, n. 12, p. $3675-3685,2010$.

MEYER, L. et al. Regeneração natural da Floresta Ombrófila Mista em Santa Catarina. In: VIBRANS, A. C. et al. (Ed.). Inventário Florístico Florestal de Santa Catarina. Blumenau: Edifurb, 2013. p. 191-222. MORISITA, M. Id-index, a measure of dispersion of individuals. Researches on Population Ecology, New York, v. 4, n. 1, p. 1-7, 1962.

NARVAES, I. S.; BRENA, D. A.; LONGHI, S. J. Estrutura da regeneração natural em Floresta Ombrófila Mista na Floresta Nacional de São Francisco de Paula, RS. Ciência Florestal, Santa Maria, v. 15, n. 4, p. 331-342, 2005.

NASCIMENTO, A. R. T.; LONGHI, S. J.; BRENA, A. D. Estrutura e padrões de distribuição espacial de espécies arbóreas em uma amostra de Floresta Ombrófila Mista em Nova Prata, RS. Ciência Florestal, Santa Maria, v. 11, n. 1, p. 105-119, 2001.

NATHAN, R.; MUELLER-LANDAU, H. C. Spatial patterns of seed dispersal, their determinants and consequences for recruitment. Trends in Ecology and Evolution, Amsterdam, v. 15, n. 7, p. 278-285, 2000. NEGRINI, M. et al. Dispersão, distribuição espacial e estratificação vertical da comunidade arbórea em um fragmento florestal no Planalto Catarinense. Revista Árvore, Viçosa, MG, v. 36, n. 5, p. 919-930, 2012.

NEGRINI, M. et al. Heterogeneidade florístico-estrutural do componente arbóreo em um sistema de fragmentos florestais no Planalto Sul catarinense. Revista Árvore, Viçosa, MG, v. 38, n. 5, p. 779-786, 2014.

OKSANEN, J. et al. Vegan: Community Ecology Package. [s. 1.: s. n], 2016. Disponível em: <http://cran.rproject.org/package=vegan>. Acesso em: 08 fev. 2016.

OLIVEIRA FILHO, A. T. et al. Delving into the variations in tree species composition and richness across South American subtropical Atlantic and Pampean forests. Journal of Plant Ecology, Oxford, v. 8, n. 3, p. 242, 2015.

PAISE, G.; VIEIRA, E. M. Produção de frutos e distribuição espacial de angiospermas com frutos zoocóricos em uma Floresta Ombrófila Mista no Rio Grande do Sul, Brasil. Revista Brasileira de Botânica, São Paulo, v. 28, n. 3, p. 615-625, 2005. 
POLISEL, R. T. et al. Structure of understory community in four stretches of Araucaria forest in the state of São Paulo, Brazil. Acta Botanica Brasilica, Belo Horizonte, v. 28, n. 1, p. 86-101, 2014.

PSCHEIDT, F. et al. Variações florístico-estruturais da comunidade arbórea associadas à distância da borda em um fragmento florestal no Planalto Sul-Catarinense. Revista Floresta, Curitiba, v. 45, n. 2, p. 421-430, 2015.

R DEVELOPMENT CORE TEAM. R: a language and environment for statistical computing. [s. 1.]: R Foundation for Statistical Computing, 2016. Disponível em: <http://www.r-project.org>. Acesso em: 08 fev. 2016.

RIBEIRO, T. M. et al. Mixed rain forest in southeastern Brazil: tree species regeneration and floristic relationships in a remaining stretch of forest near the city of Itaberá, Brazil. Acta Botanica Brasilica, Belo Horizonte, v. 27, n. 1, p. 71-86, 2013.

ROBERTS, D. W. Labdsv: ordination and multivariate analysis for ecology. [s. 1.: s. n.]: 2016. Disponível em: $<$ http://cran.r-project.org/package=labdsv>. Acesso em: 08 fev. 2016.

RONDON NETO, R. M.; WATZLAWICK, L. F.; CALDEIRA, M. V. W. Diversidade florística e síndromes de dispersão de diásporos das espécies arbóreas de um fragmento de Floresta Ombrófila Mista.

Revista Ciências Exatas e Naturais, Curitiba, v. 3, n. 2, p. 210-216, 2001.

SANTOS, K. F. et al. Regeneração natural do componente arbóreo após a mortalidade de um maciço de taquara em um fragmento de Floresta Ombrófila Mista em Lages -SC. Ciência Florestal, Santa Maria, v. 25, n. 1, p. $107-117,2015$.

SAMPAIO, M. B.; GUARINO, E. S. G. Efeitos do pastoreio de bovinos na estrutura populacional de plantas em fragmentos de Floresta Ombrófila Mista. Revista Árvore, Viçosa, MG, v. 31, n. 6, p. 1035-1046, 2007. SCHNITZER, S. A.; DALLING, J. W.; CARSON, W. P. The impacts of lianas on tree regeneration in tropical forest canopy gaps: evidence for an alternative pathway of gap-phase regeneration. Journal of Ecology, London, v. 88, n. 2, p. 655-666, 2000.

SILVA, A. C. et al. Relações florísticas e fitossociologia de uma Floresta Ombrófila Mista Montana Secundária em Lages, Santa Catarina. Ciência Florestal, Santa Maria, v. 22, n. 1, p. 193-206, 2012.

SMITH-GILL, S. J. Cytophysiological basis of disruptive pigmentary patterns in the leopardo frog, Rana pipiens. II. Wild type and mutant cell specific patterns. Journal of Morphology, Hoboken, v. 146, n. 1, p. 35-54, 1975.

SOUZA, R. P. M. et al. Estrutura e aspectos da regeneração natural de Floresta Ombrófila Mista no Parque Estadual de Campos do Jordão, SP, Brasil. Hoehnea, São Paulo, v. 39, n. 3, p. 387-407, 2012.

STONE, L.; ROBERTS, A. The checkerboard score and species distributions. Oecologia, New York, v. 85, n. 1, p. 74-79, 1990.

VIEIRA, D. L. M.; SCARIOT, A. Principles of natural regeneration of tropical dry forests for restoration. Restoration Ecology, Hoboken, v. 14, n. 1, p. 11-20, 2006.

VOLPATO, M. M. L. Regeneração natural em uma floresta secundária no domínio de Mata Atlântica: uma análise fitossociológica. 1994. 123 f. Dissertação (Mestrado em Ciência Florestal) - Universidade Federal de Viçosa, Viçosa, MG, 1994.

WEBB, C.O.; PEART, D. R. Seedling density dependence promotes coexistence of Bornean rain forest trees. Ecology, Hoboken, v. 80, n. 6, p. 2006-2017, 1999.

WHITMORE, T. C. Canopy gaps and the two major groups of forest trees. Ecology, Hoboken, v. 70, n. 3, p. 536-538, 1989.

WRIGHT, S. J. et al. Gap-dependent recruitment, realized vital rates, and size distributions of tropical trees. Ecology, Hoboken, v. 84, n. 12, p. 3174-3185, 2003. 